\title{
A REVIEW ON RECENT DEVELOPMENTS FOR THE CURE OF SALMONELLA ENTERICA SEROVAR TYPHI, THE CAUSATIVE AGENT FOR TYPHOID FEVER.
}

\author{
Muqadas Kanwal \\ Department of Microbiology and Molecular Genetics, University of Okara, Pakistan \\ Fadia Waheed \\ Institute of Microbiology and Molecular Genetics, University of the Punjab, Lahore, Pakistan \\ Hafsa Shahzadi \\ University of veterinary and Animal Sciences Lahore, Pakistan \\ Muhammad Shahbaz \\ Drug Testing Laboratory, Multan, Pakistan \\ Ahsan Noor \\ Government College University, Faisalabad, Pakistan \\ *Corresponding author \\ Email: Fadia.waheed1@gmail.com
}

\begin{abstract}
Salmonella enterica typhi is typhoid or enteric fever agent which is a serious water-borne disease and is a human host restricted organism. So, an important cause of death in underdeveloped countries, typhoid fever is a public health concern. Worldwide, 15-30 million people suffer from this disease every year, causing more than 200,000 deaths. However, several lines of evidence suggest that the advent of multidrug-resistant non-typhoidal strains of Salmonella has an important impact on the effectiveness of current strategies, including reductions in the effectiveness of early empirical treatment for controlling and managing foodborne diseases. Recent studies show more than 2000 strains of salmonella bacteria with around 100 strains connected to human infection with myriad common strains from Salmonella Heidelberg to Typhimurium to Salmonella infantis. The multi-medicinal strain S. Typhi H58 has developed into the main circulating strain in many parts of the world, and an extensively drug-resistant (XDR) subclade has been recently found. Most of the people agree that the most effective way to control infection is to vaccinate susceptible populations. The commercially available live attenuated (Ty21a) vaccine, on the other hand, is not recommendable for children under the age of six, whereas the poor long-term efficacy of Vipolysaccharide-based vaccine against typhoid fever. Furthermore, there are no vaccines available to protect against $S$. para typhi infection. Subsequently, a new formulation is urgently needed that can provide long-term protection against both pathogens while healthy for all age groups. Pakistan is the first country in the world to incorporate the WHO-recommended conjugate vaccine into its routine typhoid immunization program (2019). As a result, the purpose of this review is to describe the various diagnostic procedures for typhoid fever diagnosis and cure development. This article
\end{abstract}


addressed some of the elements and components required for the implementation of typhoid vaccine. With an analysis of past and current enteric fever vaccines in progress as well as the ethical issues relevant to CHIM in typhoid vaccine efficacy research, we have combined the new methods to predict typhoid burden and vaccines impact.

Key words: Salmonella typhi, typhoid fever, typhoid vaccination

\section{Introduction}

Salmonella enterica subspecies enterica serovar typhi, a human-specific pathogen that cause Typhoid fever, is a Gram-negative, flagellated rod-shaped, aerobic bacterium. Among the 2,300 serovars of S. enterica subspecies, $S$. typhi is unique in that it has evolved solely for infection of humans. Given its human-specificity, S. typhi could be even earlier than the split several million years ago, between humans and higher primates, or even as recent as the 50,000-100,000-year-old increase in anatomically modern humans from Africa. Approximately 140 million years ago, S. enterica and Escherichia coli shared a common ancestor. Between rRNA genes, rearrangements are caused by homologous recombination and because of this, the gene order of $S$. typhi also becomes variable. The information summarized above shows that $S$. typhi has multiple genetic variants, but it does not provide information on its age. $S$. Typhi causes 9.9-24.2 million deadly infections each year, with manifestations of disease causing 75,000-208,000 deaths worldwide (Kidgell et al., 2002; Crump et al., 2015).

S. typhi is the causative agent of typhoid fever which is a systemic febrile illness and can be transmitted by the fecal-oral route, mainly through the consumption of contaminated water and food that is also contaminated with pathogens. S. typhi causes disease by invading the mucosal surface (intestine), which then spreads to the spleen followed by pancreas, liver and bone marrow. A non-specific fever is a common side effect of the infection, which rises during the first week of illness before stabilizing in the second week with temperatures around 39-40 degrees Celsius. Acute illness is described by prolonged fever, headache, loss of craving, nausea and constipation, and at some points diarrhea (Lee et al., 2016). Different entanglements can prompt deadly outcomes whenever analyzed late or not treated successfully (Parry, 2002). Due to ineffective diagnostic laboratory capacity to differentiate typhoid fever from other febrile conditions that is a huge challenge to the developing countries regarding to public health (Tau $e t$ al., 2017). Typhoid patients in endemic areas are treated as outpatients with oral antibiotics, with hospitalization required only in the most serious cases. Deterioration that may complicate disease and chronic fecal carriage that can lead to continuous transmission in typhoid cases are two major concerns (Parry \& Beeching, 2009). Chronic S. typhi infection is historically associated with long-term bacterial excretion and is commonly located on the Gall bladder (Young et al., 2002). Epidemiological studies conducted in S. Typhi endemic regions like Bolivia and Ecuador and some affected regions of Korea, India, Japan, Pakistan, have revealed that approximately $90 \%$ of chronically infected carriers also have gallstones, indicating that, for gallbladder cancer (GC)this association is a major predisposing factor (Ochman \& Wilson, 1987; Caygill et al., 1994; Liu \& Sanderson, 1995).

Typhoid fever was traditionally treated with 2-3 weeks of chloramphenicol in the late 1980's. The number of plasmid mediated MDR (multidrug resistance) strains to chloramphenicol, cotrimoxazole and ampicillin has since been increasing. Following the emergence of MDR strains, fluoroquinolones (ciprofloxacin and ofloxacin) have 
become the preferred drug (Kalra et al., 2003). Now a days, to diagnose Typhoid fever, a combination of clinical symptoms, isolation of Salmonella typhi from body fluids, and the Widal test (Echeita \& Usera, 1998; Willke et al., 2002; Lee et al., 2016).

The WHO Strategic Immunization Advisory Group (SAGE) 2017 proposed the implementation of typhoid conjugate (TCV) vaccines for children aged 6 months or older in typhoid-endemic countries. This will possibly boost further our understanding not only at national and sub-national level of the typhoid burden, but also at world level (Organization, 2014). According to most recent estimates of the World Health Organization (WHO) 2019, worldwide there are between 11 million and 21 million cases, 128,000 to 161,000 typhoid deaths a year (Gibani et al., 2018). Typhi has 22 million typhoid fatalities, 5.4 million paratyphoid fever cases and 216,000 deaths worldwide each year (Crump et al., 2004). About 1-4\% of the affected people are chronic asymptomatic carriers in the areas where S. Typhi is prevalent, posing a risk to local public health (Sippel et al., 1989; Parry, 2002).

\section{Vaccine Developments in Cure of Salmonella}

In recent decades, there have been several improvements in typhoid fever in accordance with the understanding of its global burden, including the geographical representation of high-quality incidence studies of typhoid fever and an increase in the number of cases, as well as greater complexity of modelling approaches. There are several barriers to the generation of evidence to promote the implementation, however, of an effective S. Typhi vaccine in endemic countries worldwide. With a no reliable point-of-care diagnostic, nonspecific clinical presentation and predicting the precise ailment and thus the need for vaccination to prevent dumps, loss of life, and financial burden has been difficult (Ibe et al., 2019).

\section{Multiple Drugs Resistant}

The antimicrobial arsenal gradually exhausted due to drug resistant strains, making treatment difficult and costly. A new extensive antibiotic resistant strain (XDR) emerged in Hyderabad in 2016, infecting more than 8,000 people in 14 areas and has since been distributed across Sindh. A recent outbreak of XDR typhoid (defined as MDR plus fluoroquinolone resistance) has been reported in Pakistan (Klemm et al., 2018) These XDR Salmonella isolates are not only resistant to first-line antibiotics (trimethoprim-sulfamethoxazole, chloramphenicol and ampicillin), they are also resistant to ciprofloxacin. They do not respond to cephalosporins (third generation antibiotics) due to the acquisition of the H58 haplotype mutation. In a large-scale outbreak in Hyderabad, Sindh, for the first time, the new S. typhi XDR clone isolated. The chromosomally located resistance region is present in this $S$. typhi along the IncY plasmid, which contains the blaCTX-M15 extended-spectrum -lactamase, which mediates ceftriaxone resistance, as well as the qnrS fluoroquinolone resistance gene. S. typhi can convert from MDR to XDR in one single phase by acquiring a plasmid which can propagate the XDR clone globally. This problem must also be addressed with diagnostic and therapeutic options. In every clinical setting, these include a quick and accurate diagnosis of typhoid fever. Widal and Typhidot tests, are unreliable and obsolete serological tests that must be phased out. We need 3rd generation cephalosporins and IV ceftriaxone for empirical therapy and for serious cases, respectively (Andualem $e t$ al., 2014). 
Oral cefixime (high dose) should be used in casualty settings in less serious situations, including MDR typhoid. Sensitive strains can be treated safely with oral administration of Amoxicillin or Trimethoprimsulfamethoxazole. Treatment must last for at least 10-14 days or until the patient is afebrile for at least 5 days, whichever comes first. With XDR typhoid, the fear of the dreadful "untreatable typhoid" has become a hideous fact. For uncomplicated XDR typhoid, azithromycin is currently the only effective first-line oral treatment option. Carbapenems are the only IV option for patients with suspected severe or complicated XDR typhoid fever (Ryan $e t$ al., 2019).The provincial government took some preventative measures, such as other things (Khan, 2019).

Antibiotic resistance is a global health emergency that is accelerated by the overuse of antibiotics (Fatima $e t$ al., 2019). To prevent further outbreaks, a new typhoid vaccine has been approved for addition to the Extended Program for Immunization (Fatima et al., 2019). The Federal EPI/ Ministry of NHSR \& C also approved the first new conjugate typhoid vaccine (TCV) for routine EPI schedule at the national level. The vaccine (Typbar-TCV®) was prequalified by the WHO has a number of advantages, including the ability to start treatment at the age of six months, a protected efficacy of 87 percent, and a protection period of more than five years (Khan, 2019).

\section{Vi-TT and Vi-TCV Vaccines}

In Oxford, UK, a controlled human infection model (CHIM) was created to quicken vaccine research, both with S. Typhi and S. Para typhi (Waddington et al., 2014). A series of candidate vaccines, including the World Health Organisation's latest Vi polysacharide-proteine conjugate (Vi-TT) typhoid, have been adequately validated using this model (Burki, 2018). The data supported the Immunization (SAGE) recommendation suggested by the WHO Strategic Advisory Group of Experts in 2017 regarding the use of TCVs (typhoid conjugate vaccines) in countries with heavy, as well as the Gavi commitment to fund the typhoid conjugate vaccines administration for children older than 6 months in typhoid-endemic countries (Organization, 2017). For 2019-2020, Gavi announced a window of $\$ 85$ million supporting the implementation of TCVs in low-income countries based on the SAGE recommendation (Meiring et al., 2018). In December 2017, WHO accepted the Typbar-TCV Prequalification to easily be introduced in low-income countries (Meiring et al., 2019). Bharat Biotech International, Hyderabad, India, has developed a new first-generation typhoid Vi conjugate vaccine (Typbar-TCV). TCVs primarily made by conjugation of 25 grams of VI capsular polysaccharide with a more complex protein. This conjugation make TCVs efficient as it can stimulate parts of the immune system that VI polysaccharide vaccines fails to do. As a result, a broader age range will develop stronger, longer-lasting immunity. Vi-safety TCV's and immunogenicity have been studied in children as young as six months old as well as adults in India, and the virus is extremely immunogenic. TCVs will affect AMR infections in the same way that susceptible typhoid will, according to our modelling article (Mohan et al., 2015; Kaufhold et al., 2019).

\section{Benefits of TCVS (Typhoid conjugate vaccine)}

New TCVs can overcome the limitations of past vaccines. These vaccines provide immunity for longer period in fewer doses and can be given to children as early as in infancy, making them suitable for use in routine childhood immunization programs. Increased use of TCVs through routine vaccination will minimize the use of antibiotics and slow the spread of drug-resistant bacteria. Typbar-TCV is protected, well tolerated, and provide immunity long lasting 
and strong response in all age groups for longer than the presently approved polysaccharide typhoid vaccine (Mohan et al., 2015).

Adults, children, and infants over the age of 6 months receive at least 3 years of protection from a single 0.5 $\mathrm{mL}$ dose of Typbar-TCV injected intramuscularly. More research is needed to determine the duration of protection and whether booster doses are required. Typbar-TCV's high immunogenicity makes it an excellent candidate in typhoid endemic countries, particularly for children under the age of two, who are particularly susceptible to typhoid fever. Oxford University researchers used a controlled human infection model (CHIM) conducting the first clinical trial to determine Typbar-TCV effectiveness. The vaccine decreased the number of typhoid cases by half and was efficient by 87.1 percent when fever endpoints $>38^{\circ} \mathrm{C}$ accompanied by a positive blood culture. Surprisingly, this estimate matched the results of a serological analysis of protection in an immunogenicity trial in India. Aforementioned data provided critical support for global policy decisions (Jamka et al., 2019).

\section{Recommended Vaccines}

Currently three recommended typhoid vaccines are used. The first one is the injectable typhoid conjugate vaccine (TCV), which is made up of ViCPS linked to the tetanus protein which is approved for below year and up to 45 years aged group public. The second is an un-conjugated injectable polysaccharide vaccine based on purified VI antigen (Vi-PS) for people over the age of two years. The third vaccine is approved for six years of age is an oral liveattenuated Ty21a vaccine involve in capsule formulation. It is suitable for use in younger children and expects a longer safety period due to its improved immunological characteristics while TCV is preferred at all ages (Martin, 2012; Organization, 2019).

\section{Current Typhoid Vaccines}

Current Typhoid Vaccines are characterized as Vi-capsular polysaccharide (ViCPS) injectable vaccine and live attenuated Ty21a (oral) vaccine, both are worldwide accessible. Ty21a is standard to use for six years and up to this aged group children with three to four doses recommendation. The ViCPS vaccine is approved for children under the age of two. And in older, after every two to three years a booster dose required (Rebaza \& Lee, 2015).

Parts of the immune system are stimulated by the newly available typhoid conjugate vaccines (TCVs) that the Vi polysaccharide vaccines cannot, thus inducing longer-lasting immunity, robust among a broader age range (Puttarajanna et al.; Andrews et al., 2019).

\section{Typhoid Vaccines: Current Status}

Before 2017, there were two types of typhoid vaccines globally obtainable: one is an oral which is liveattenuated named as Ty21a vaccine for given to the children of 6 years age groups and other is injectable capsular polysaccharide vaccine named as ViCPS vaccine given to the 2 years of age children, with only the latter being WHO pre-qualified. Even though typhoid's significant and harmful impact, WHO's recommended its use, these vaccines are underutilized in high-burden countries. Because neither of these vaccines is approved for children under the age of two or subsidized by Gavi, but in regular immunization programs in low-resource countries it was hard to include them. In world, Pakistan reported as the first country to introduce the WHO-recommended conjugate vaccine (TCVS) 
into its routine typhoid vaccination program today (2019). The anti-typhoid vaccine has been administered to nine million children in Sindh during began on November 18 (Bhuiyan et al., 2014).

\section{Conclusion}

With the increasing number of cases, there is an urgent need for antibiotic resistance to be brought to light to promote rigorous research for the development of newer drugs to combat resistant typhoid strains and say no to antibiotics for a minor disease that causes increasing resistance day by day. This article also shows the development of high-quality TCV introduction. advocacy of TyVAC. This study involves improvement of the health of some of the most vulnerable people worldwide through the implementation of TCVs where it is most required to reduce typhoid burden significantly and in clinically meaningful way.

\section{References}

Andrews, J. R., Baker, S., Marks, F., Alsan, M., Garrett, D., Gellin, B. G., Saha, S. K., Qamar, F. N., Yousafzai, M. T.\& Bogoch, I. I. 2019. Typhoid conjugate vaccines: a new tool in the fight against antimicrobial resistance. The Lancet Infectious Diseases, 19: e26-e30.

Andualem, G., Abebe, T., Kebede, N., Gebre-Selassie, S., Mihret, A. \& Alemayehu, H. 2014. A comparative study of Widal test with blood culture in the diagnosis of typhoid fever in febrile patients. BMC Research Notes, 7: 1-6.

Bhuiyan, T. R., Choudhury, F. K., Khanam, F., Saha, A., Sayeed, M. A., Salma, U., Lundgren, A., Sack, D. A., Svennerholm, A.-M. \& Qadri, F. 2014. Evaluation of immune responses to an oral typhoid vaccine, Ty21a, in children from 2 to 5 years of age in Bangladesh. Vaccine, 32: 1055-1060.

Burki, T. 2018. Typhoid conjugate vaccine gets WHO prequalification. The Lancet Infectious Diseases, $18: 258$.

Caygill, C. P., Hill, M. J., Braddick, M. \& Sharp, J. C. 1994. Cancer mortality in chronic typhoid and paratyphoid carriers. The Lancet, 343: 83-84.

Crump, J. A., Sjölund-Karlsson, M., Gordon, M. A. \& Parry, C. M. 2015. Epidemiology, clinical presentation, laboratory diagnosis, antimicrobial resistance, and antimicrobial management of invasive Salmonella infections. Clinical Microbiology Reviews, 28: 901-937.

Crump, J. A., Luby, S. P. \& Mintz, E. D. 2004. The global burden of typhoid fever. Bulletin of the World Health Organization, 82:346-353.

Echeita, M. \& Usera, M. 1998. Chromosomal rearrangements in Salmonella enterica serotype Typhi affecting molecular typing in outbreak investigations. Journal of Clinical Microbiology, 36: 2123-2126.

Fatima, K., Shabbir, O. \& Ochani, R. K. 2019. Emergence of a highly resistant typhoid strain: a new global health challenge. Le Infezioni in Medicina, 27: 109-110.

Gibani, M. M., Britto, C. \& Pollard, A. J. 2018. Typhoid and paratyphoid fever: a call to action. Current Opinion in Infectious Diseases, 31: 440. 
Ibe, M., Odimegwu, D. \& Onuigbo, E. 2019. Alginate-coated chitosan microparticles encapsulating an oral plasmidcured live Salmonella enterica serovar Gallinarum vaccine cause a higher expression of interferon-gamma in chickens compared to the parenteral live vaccine. Avian Pathology, 48: 423-428.

Jamka, L. P., Simiyu, K. W., Bentsi-Enchill, A. D., Mwisongo, A. J., Matzger, H., Marfin, A. A., Pollard, A. J. \& Neuzil, K. M. 2019. Accelerating typhoid conjugate vaccine introduction: what can be learned from prior new vaccine introduction initiatives? Clinical Infectious Diseases, 68:S171-S176.

Kalra, S., Naithani, N., Mehta, S. \& Swamy, A. 2003. Current trends in the management of typhoid fever. Medical Journal, Armed Forces India, 59: 130-137.

Kaufhold, S., Yaesoubi, R. \& Pitzer, V. E. 2019. Predicting the impact of typhoid conjugate vaccines on antimicrobial resistance. Clinical Infectious Diseases, 68: S96-S104.

Khan, E. A. 2019. XDR Typhoid: The problem and its solution. Journal of Ayub Medical College Abbottabad, 31: $139-140$.

Kidgell, C., Reichard, U., Wain, J., Linz, B., Torpdahl, M., Dougan, G. \& Achtman, M. 2002. Salmonella typhi, the causative agent of typhoid fever, is approximately 50,000 years old. Infection, Genetics and Evolution, 2: $39-45$.

Klemm, E. J., Shakoor, S., Page, A. J., Qamar, F. N., Judge, K., Saeed, D. K., Wong, V. K., Dallman, T. J., Nair, S. \& Baker, S. 2018. Emergence of an extensively drug-resistant Salmonella enterica serovar Typhi clone harboring a promiscuous plasmid encoding resistance to fluoroquinolones and third-generation cephalosporins. MBio, 9: 12-18.

Lee, J.-S., Mogasale, V. V., Mogasale, V. \& Lee, K. 2016. Geographical distribution of typhoid risk factors in low and middle income countries. BMC Infectious Diseases, 16: 1-10.

Liu, S.-L. \& Sanderson, K. E. 1995. Rearrangements in the genome of the bacterium Salmonella typhi. Proceedings of the National Academy of Sciences, 92: 1018-1022.

Martin, L. B. 2012. Vaccines for typhoid fever and other salmonelloses. Current Opinion in Infectious Diseases, 25: 489-499.

Meiring, J. E., Laurens, M. B., Patel, P., Patel, P., Misiri, T., Simiyu, K., Mwakiseghile, F., Tracy, J. K., Masesa, C. \& Liang, Y. 2019. Typhoid vaccine acceleration consortium Malawi: a phase III, randomized, double-blind, controlled trial of the clinical efficacy of typhoid conjugate vaccine among children in Blantyre, Malawi. Clinical Infectious Diseases, 68: S50-S58.

Meiring, J. E., Patel, P., Patel, P. \& Gordon, M. A. 2018. Typhoid conjugate vaccines: making vaccine history in Africa. Expert Review of Vaccines, 17: 673-676.

Mohan, V. K., Varanasi, V., Singh, A., Pasetti, M. F., Levine, M. M., Venkatesan, R. \& Ella, K. M. 2015. Safety and immunogenicity of a Vi polysaccharide - tetanus toxoid conjugate vaccine (typbar-TCV) in healthy infants, children, and adults in typhoid endemic areas: a multicenter, 2-cohort, open-label, double-blind, randomized controlled phase 3 study. Clinical Infectious Diseases, 61:393-402.

Ochman, H. \& Wilson, A. C. 1987. Evolution in bacteria: evidence for a universal substitution rate in cellular genomes. Journal of Molecular Evolution, 26: 74-86. 
Organization, W. H. 2019. Typhoid vaccines: WHO position paper, March 2018-Recommendations. Vaccine, 37: 214-216.

Organization, W. H. 2017. WHO position on the use of fractional doses-June 2017, addendum to vaccines and vaccination against yellow fever WHO: Position paper-June 2013. Vaccine, 35: 5751-5752.

Organization, W. H. 2014. Meeting of the Strategic Advisory Group of Experts on immunization, October 2014conclusions and recommendations. Weekly Epidemiological Record-Relevé épidémiologique hebdomadaire, 89: $561-576$.

Parry, C. M. \& Beeching, N. J. 2009. Treatment of enteric fever. British Medical Journal Publishing Group, UK, pp. $1-20$.

Parry, C. M. 2002. Hien 1Tr, Douqan G, et a1. Typhoid fever. N Engl Med, 347: 1770-1782.

Puttarajanna, B., Prasad, R., Kulkarni, R., Bajaj, J., Rao, B. \& Ganguly, R. J. President's Letter.

Rebaza, A. \& Lee, P. J. 2015. One more shot for the road: A review and update of vaccinations for pediatric international travelers. Pediatric Annals, 44: e89-e96.

Ryan, E. T., Andrews, J. \& John, J. 2019. Treatment and prevention of enteric (typhoid and paratyphoid) fever. UpToDate. Waltham, UpToDate Inc. http://www. uptodate. com.

Sippel, J., Bukhtiari, N., Awan, M., Krieg, R., Duncan, J., Karamat, K., Malik, I., Igbal, L. \& Legters, L. 1989. Indirect immunoglobulin $\mathrm{G}$ (IgG) and IgM enzyme-linked immunosorbent assays (ELISAs) and IgM capture ELISA for detection of antibodies to lipopolysaccharide in adult typhoid fever patients in Pakistan. Journal of Clinical Microbiology, 27: 1298-1302.

Tau, N. P., Smith, A. M., Wain, J. R., Tarupiwa, A., Coulibaly, K. J. \& Keddy, K. H. 2017. Development and evaluation of a multiple-locus variable-number tandem-repeats analysis assay for subtyping Salmonella typhi strains from sub-Saharan Africa. Journal of Medical Microbiology, 66: 937-945.

Waddington, C. S., Darton, T. C., Jones, C., Haworth, K., Peters, A., John, T., Thompson, B. A., Kerridge, S. A., Kingsley, R. A. \& Zhou, L. 2014. An outpatient, ambulant-design, controlled human infection model using escalating doses of Salmonella typhi challenge delivered in sodium bicarbonate solution. Clinical Infectious Diseases, 58: 1230-1240.

Willke, A., Ergonul, O. \& Bayar, B. 2002. Widal test in diagnosis of typhoid fever in Turkey. Clinical and Diagnostic LaboratoryImmunology, 9: 938-941.

Young, D., Hussell, T. \& Dougan, G. 2002. Chronic bacterial infections: living with unwanted guests. Nature immunology, 3: 1026-1032. 